\title{
Sur le nombre de Eojasiewicz à l'infini d'un polynôme
}

\author{
par Pierrette Cassou-Noguès (Talence) et Ha Huy Vui (Hanoi)
}

Résumé. Soit $f$ un polynôme à deux indéterminées. On appelle nombre de Eojasiewicz à l'infini de $f$ le nombre de Łojasiewicz à l'infini de son application gradient. Dans cet article nous montrons tout d'abord que l'on peut calculer le nombre de Łojasiewicz d'un polynôme à partir des diagrammes de Eisenbud et Neumann de toutes les courbes $f(x, y)=$ $t$. Ensuite nous montrons que l'on peut définir un nombre de Łojasiewicz intrinsèque en prenant le maximum des nombres de Łojasiewicz de $f \circ \phi$ si $f$ est bon et le minimum des nombres de Łojasiewicz de $f \circ \phi$ sinon, lorsque $\phi$ parcourt les automorphismes de $\mathbb{C}^{2}$. On donne un exemple où l'on ne peut pas trouver un automorphisme de $\mathbb{C}^{2}$ qui réalise à la fois le degré, le nombre de points à l'infini et le nombre de Łojasiewicz intrinsèques. On montre que si $f$ est non dégénéré pour son polygone de Newton, ou satisfait les conditions de Oka, alors le degré, le nombre de points à l'infini et le nombre de Łojasiewicz sont le degré, le nombre de points à l'infini et le nombre de Łojasiewicz intrinsèques.

Soit $f\left(x_{0}, \ldots, x_{n}\right)=0$ une équation pour un germe d'hypersurface à singularité isolée $\left(X_{0}, 0\right) \subset\left(\mathbb{C}^{n+1}, 0\right)$. Le plus petit exposant $\theta$ tel qu'il existe un voisinage $U$ de 0 dans $\mathbb{C}^{n+1}$ et une constante $C_{2}$ tels que

$$
\|\operatorname{grad} f(x)\| \geq C_{2}\|x\|^{\theta}
$$

est appelé le nombre de Eojasiewicz du germe $\left(X_{0}, 0\right)$. Teissier [T] a montré que c'est le plus grand des invariants polaires associés au germe, et pour les courbes, $[\mathrm{LMW}]$ ont prouvé que l'on peut le calculer à l'aide du diagramme de Eisenbud et Neumann de l'entrelacs algébrique défini par le germe. Lichtin [Li], toujours dans le cas des courbes, a exprimé le nombre de Łojasiewicz à l'aide du polygone de Newton de $f$, lorsque $f$ est non dégénéré.

Soit $f$ un polynôme à deux indéterminées. On définit le nombre de Łojasiewicz à l'infini de $f$ par

$$
\mathcal{L}_{\infty}(f)=\lim _{r \rightarrow \infty} \frac{\ln \phi(r)}{\ln r} \quad \text { où } \quad \phi(r)=\inf _{\|x\|=r}\|\operatorname{grad} f(x)\| .
$$

Nous allons tout d'abord montrer dans cet article que l'on peut exprimer

1991 Mathematics Subject Classification: 14E05, 14F45.

Key words and phrases: Lojasiewicz number at infinity. 
$\mathcal{L}_{\infty}(f)$ à l'aide de tous les diagrammes de Eisenbud et Neumann des courbes $f(x, y)=t$.

Le nombre de Łojasiewicz à l'infini dépend du système de coordonnées. En effet [CK1],

$$
\mathcal{L}_{\infty}(f)= \begin{cases}p-1 & \text { pour } f(x, y)=y^{p}+x^{p}, \\ -1+p / q & \text { pour } f\left(x+y^{q}, y\right)=y^{p}+\left(x+y^{q}\right)^{p} .\end{cases}
$$

D'autres nombres associés à $f$ varient de la même façon : le degré et le nombre de points à l'infini. Neumann $[\mathrm{N}]$ a introduit la notion de degré intrinsèque de $f$, comme étant le minimum des degrés de $f \circ \phi$ pour tous les $\phi \in \operatorname{Aut}\left(\mathbb{C}^{2}\right)$, et de nombre de points à l'infini intrinsèque de $f$, comme étant le maximum du nombre de points à l'infini de $f \circ \phi$ (ce maximum existe car le nombre de points à l'infini est borné par le nombre de places à l'infini qui est invariant).

Nous allons montrer que l'on peut définir un nombre de Eojasiewicz à l'infini intrinsèque de la façon suivante :

$$
\mathcal{L}_{\infty, \text { int }}(f)= \begin{cases}\max _{\phi \in \operatorname{Aut}\left(\mathbb{C}^{2}\right)} \mathcal{L}_{\infty}(f \circ \phi) & \text { si } f \text { est bon, } \\ \inf _{\phi \in \operatorname{Aut}\left(\mathbb{C}^{2}\right)} \mathcal{L}_{\infty}(f \circ \phi) & \text { si } f \text { n'est pas bon. }\end{cases}
$$

Enfin, nous étudions le problème de l'existence d'un automorphisme privilégié pour un polynôme, c'est-à-dire de l'existence d'un automorphisme $\phi$ tel que

$$
\operatorname{deg}_{\text {int }}(f)=\operatorname{deg}(f \circ \phi), \quad n_{\infty, \text { int }}(f)=n_{\infty}(f \circ \phi), \quad \mathcal{L}_{\infty, \text { int }}(f)=\mathcal{L}_{\infty}(f \circ \phi) .
$$

Nous donnons un exemple qui montre qu'un tel automorphisme n'existe pas en général et nous prouvons que si $f$ est non dégénéré pour son polygone de Newton à l'infini, ou si $f$ satisfait les obstructions de Oka $[\mathrm{O}]$, alors

$$
\operatorname{deg}_{\text {int }}(f)=\operatorname{deg}(f), \quad n_{\infty, \text { int }}(f)=n_{\infty}(f), \quad \mathcal{L}_{\infty, \text { int }}(f)=\mathcal{L}_{\infty}(f) .
$$

Nous démontrons un analogue du théorème de Lichtin pour le nombre de Łojasiewicz à l'infini.

Une grande partie de ce travail est basé sur l'étude des diagrammes de Eisenbud et Neumann à l'infini d'une courbe et sur le lien avec le polygone de Newton à l'infini du polynôme qui définit la courbe. Dans la première partie nous rappelons ce que nous utilisons sur les diagrammes de Eisenbud et Neumann.

\section{Rappels sur les diagrammes de Eisenbud et Neumann}

1. Diagramme associé à une courbe $V \subset \mathbb{C}^{2}$. Soit $f: \mathbb{C}^{2} \rightarrow \mathbb{C}$ une application polynomiale. L'entrelacs à l'infini d'une courbe $V$ définie par $f=t$ peut se représenter par un diagramme de Eisenbud et Neumann, qui donne une façon de coder les singularités de la courbe en ses points d'intersection avec la droite à l'infini. Pour expliquer comment obtenir le 
diagramme à l'infini d'une courbe, il faut déjà rappeler comment on construit le diagramme de Eisenbud et Neumann d'un germe de courbe algébrique [EN].

Si $f$ est un germe de courbe plane, l'intersection de $f^{-1}(0)$ avec une petite sphère de rayon $\varepsilon$ centrée à l'origine est un entrelacs appelé entrelacs algébrique de $f$. Son diagramme de Eisenbud et Neumann s'obtient de la façon suivante : Si l'on a une seule branche, on écrit son développement de Puiseux sous la forme

$$
y=x^{q_{1} / p_{1}}\left(a_{1}+x^{q_{2} /\left(p_{1} p_{2}\right)}\left(a_{2}+\ldots+\left(a_{s-1}+a_{s} x^{q_{s} /\left(p_{1} p_{2} \ldots p_{s}\right)}\right) \ldots\right)\right) .
$$

L'entrelacs algébrique correspondant a pour diagramme

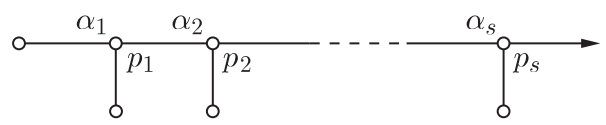

avec

$$
\alpha_{1}=q_{1}, \quad \alpha_{j+1}=q_{j+1}+p_{j} p_{j+1} \alpha_{j}, \quad j \geq 1 .
$$

Si l'on a une autre branche

$$
y=x^{q_{1}^{\prime} / p_{1}^{\prime}}\left(a_{1}^{\prime}+x^{q_{2}^{\prime} /\left(p_{1}^{\prime} p_{2}^{\prime}\right)}\left(a_{2}^{\prime}+\ldots+\left(a_{r-1}^{\prime}+a_{r}^{\prime} x^{q_{r}^{\prime} /\left(p_{1}^{\prime} p_{2}^{\prime} \ldots p_{r}^{\prime}\right)}\right) \ldots\right)\right),
$$

on suppose que pour $i \leq n, p_{i}^{\prime}=p_{i}, q_{i}^{\prime}=q_{i}$ et $a_{i}^{\prime}=a_{i}$. On a trois cas à considérer.

Tout d'abord, si $n=r$ ou $n=s$, on a

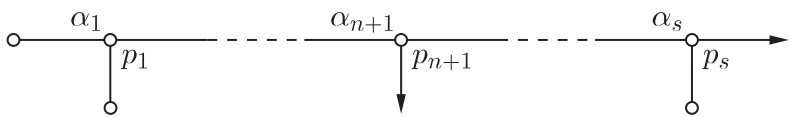

Si $r>n$ et $s>n$, et $q_{n+1} / p_{n+1}=q_{n+1}^{\prime} / p_{n+1}^{\prime}$, on a

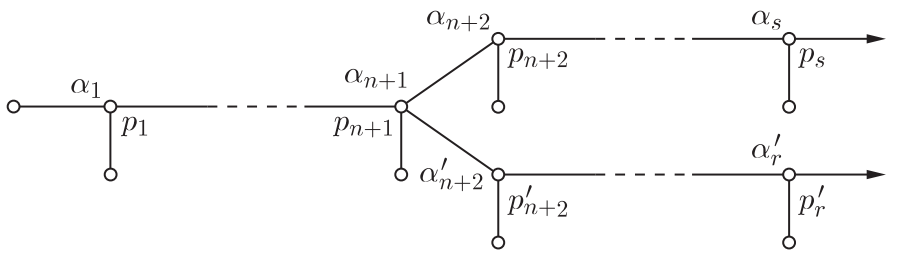

Sinon, $q_{n+1} / p_{n+1} \leq q_{n+1}^{\prime} / p_{n+1}^{\prime}$; dans ce cas, on a 


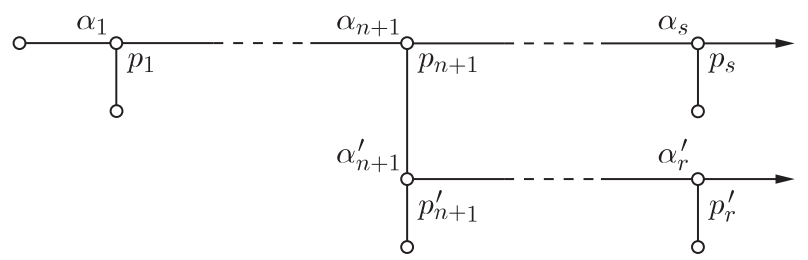

Si l'on a une troisième branche, on procède de la même façon en tenant compte des 2 branches précédentes. Si $f=f_{1}^{m_{1}} f_{2}^{m_{2}} \ldots f_{r}^{m_{r}}$, on obtient un multientrelacs que l'on représente à partir des précédents en mettant $\left(m_{i}\right)$ en face de la flèche correspondante. Si maintenant on considère une singularité qui n'est pas à l'origine, on fait un changement de variable pour s'y ramener.

Pour l'entrelacs à l'infini de $V$, on considère les zéros de la forme homogène de plus haut degré de $f$. Pour chacun de ces zéros $\left(a_{i}, b_{i}\right)$, on considère le diagramme de Eisenbud et Neumann de l'entrelacs algébrique de $F(x, 1, z)$ au point $\left(a_{i} / b_{i}, 0\right)$ si $b_{i}$ est non nul, ou $F(1, y, z)$ au point $(0,0)$ si $b_{i}=0$ où $F(x, y, z)$ est le polynôme homogène tel que $F(x, y, 1)=f(x, y)$. On écrit les mêmes diagrammes de Eisenbud et Neumann en remplaçant $\left(p_{1}, \alpha_{1}\right) \operatorname{par}\left(p_{1}, p_{1}-\alpha_{1}\right)$ et $\left(p_{i}, \alpha_{i}\right)$ par $\left(p_{i}, p_{1}^{2} p_{2}^{2} \ldots p_{i-1}^{2} p_{i}-\alpha_{i}\right)$. On câble ensuite les diagrammes obtenus par les composantes convenables.

EXemple I. On considère la courbe $V$ définie par $f=0$ où

$$
\begin{aligned}
f= & \left(x^{2} y+1\right)^{4} y^{4}+x^{11} y^{5}+x^{13} y^{2}+x^{14}-4\left(x^{2} y+1\right)^{3} y^{3} \\
& +6\left(x^{2} y+1\right)^{2} y^{2}-4\left(x^{2} y+1\right) y-x^{2} y .
\end{aligned}
$$

$f$ est un polynôme de degré 16 . La forme homogène de plus haut degré est $x^{8} y^{8}+x^{11} y^{5}=x^{8} y^{5}\left(x^{3}+y^{3}\right)$. On a donc 5 points à l'infini. Seuls les points $(1,0,0)$ et $(0,1,0)$ sont des points singuliers. On obtient

$$
\begin{aligned}
F(x, y, z)= & \left(x^{2} y+z^{3}\right)^{4} y^{4}+x^{11} y^{5}+x^{13} y^{2} z+x^{14} z^{2}-4\left(x^{2} y+z^{3}\right)^{3} y^{3} z^{4} \\
& +6\left(x^{2} y+z^{3}\right)^{2} y^{2} z^{8}-4\left(x^{2} y+z^{3}\right) y z^{12}-x^{2} y z^{13} \\
F(x, 1, z)= & \left(x^{2}+z^{3}\right)^{4}+x^{11}+x^{13} z+x^{14} z^{2}-4\left(x^{2}+z^{3}\right)^{3} z^{4} \\
& +6\left(x^{2}+z^{3}\right)^{2} z^{8}-4\left(x^{2}+z^{3}\right) z^{12}-x^{2} z^{13} .
\end{aligned}
$$

On voit que le germe de $F(x, 1, y)$ au point $(0,0)$ est un germe de courbe irréductible dont les paires de Puiseux successives sont $(2,3),(1,2),(4,1)$. Le diagramme de l'entrelacs algébrique est donc

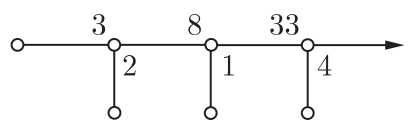


Ensuite,

$$
\begin{aligned}
F(1, y, z)= & \left(y+z^{3}\right)^{4} y^{4}+y^{5}+y^{2} z+z^{2}-4\left(y+z^{3}\right)^{3} y^{3} z^{4} \\
& +6\left(y+z^{3}\right)^{2} y^{2} z^{8}-4\left(y+z^{3}\right) y z^{12}-y z^{13}
\end{aligned}
$$

Le germe à l'origine de $F(1, y, z)$ a 2 branches, de paires de Puiseux $(3,1)$ et $(2,1)$. Donc le diagramme de l'entrelacs algébrique est

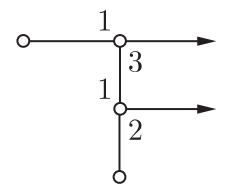

Finalement, le diagramme de Eisenbud et Neumann de l'entrelacs à l'infini de $f(x, y)=0$ est

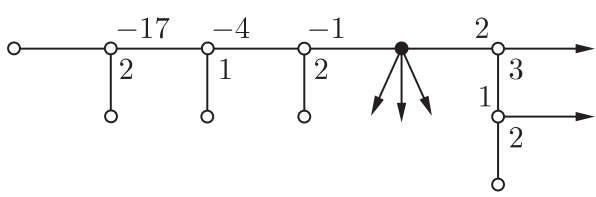

On dit que le diagramme de Eisenbud et Neumann construit de cette façon est le diagramme associé à la courbe $V$. Le sommet qui a servi à joindre les diagrammes de chaque point à l'infini s'appelle la racine du diagramme.

On a deux types de sommets, les flèches et les autres. On note $\mathcal{F}$ l'ensemble des flèches et $\mathcal{V}$ l'ensemble des sommets qui ne sont pas des flèches. Pour chaque élément $v \in \mathcal{V}$, on appelle valence et on note $\delta_{v}$ le nombre de cotés qui convergent vers $v$.

A chaque $v \in \mathcal{V}$, on associe une composante virtuelle $[\mathrm{N}]$ (encore appelée compagnon intrinsèque [LMW]), notée $S_{v}$. On note alors $l_{v}=\mathcal{L}\left(S_{v}, L\right)$ le coefficient d'enlacement de cette composante virtuelle avec l'entrelacs $L$. On calcule ces coefficients de la façon suivante :

LEMME 1 [EN, Section 10]. Le coefficient d'enlacement d'une composante avec une composante de multiplicité $m$ de l'entrelacs est le produit de tous les entiers adjacents au chemin du diagramme qui joint ces composantes (qui ne sont pas sur le chemin) et de $m$.

Soit $f: \mathbb{C}^{2} \rightarrow \mathbb{C}$ une application polynomiale. Une fibre $f^{-1}(t)$ est dite régulière s'il existe un voisinage $D$ de $t$ et un sous-ensemble compact $K$ de $\mathbb{C}^{2}$ tel que $\left.f\right|_{f^{-1}(D)-K}$ soit une fibration localement triviale.

Neumann [N, Prop. 5.4] a montré que si $f^{-1}(t)$ est régulière, alors $l_{v} \geq 0$ pour tout $v \in \mathcal{V}$, et Ha [H, Theorem 2.4.2] et Le Van Thanh et Neumann [LN, Theorem 1.2] ont démontré la réciproque. 
Neumann a encore démontré

ThÉORÈme 2 [N, p. 450]. Une courbe algébrique réduite et un plongement de $\mathbb{C}^{2} \subset \mathbb{P}^{2}$ déterminent un diagramme associé à l'entrelacs à l'infini de $V$. Le nombre de points à l'infini $n_{\infty}(V)$ de $V$ est la valence de la racine du diagramme associé, et le degré de la courbe est $l_{\mathrm{rac}}$, le nombre d'enlacement de la composante virtuelle à la racine, avec l'entrelacs.

2. Diagramme minimal associé à une courbe $V \subset \mathbb{C}^{2}$. Neumann $[\mathrm{N}$, p. 470] introduit la notion de diagramme minimal. On dit qu'un diagramme $\Omega$ est minimal si c'est l'un des diagrammes

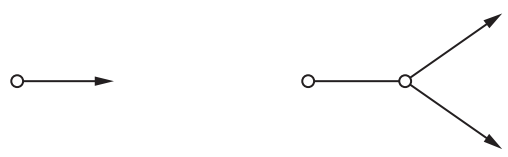

ou s'il est de la forme

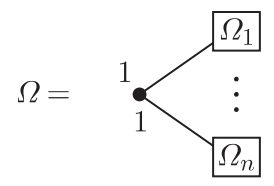

où chaque diagramme $\Omega_{i}$ est minimal, et si $n=1, \Omega$ n'est pas de la forme

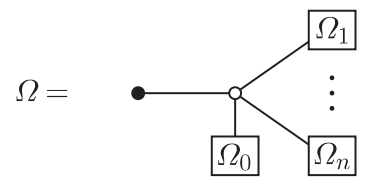

avec $q>1$.

On rend les diagrammes $\Omega_{i}$ minimaux en

1) enlevant tout côté de la forme

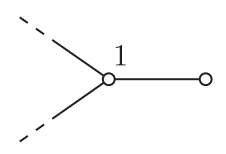

ainsi que le sommet à sa droite;

2) remplaçant deux côtés

par un seul et en supprimant le sommet; 
3) remplaçant

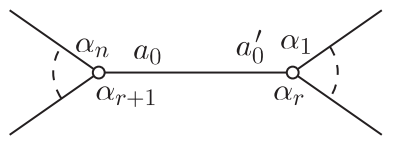

où $a_{0} a_{0}^{\prime}-\alpha_{1} \ldots \alpha_{r} \alpha_{r+1} \ldots \alpha_{n}=0$ par

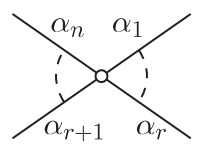

Exemples. 1) Le diagramme minimal de l'exemple I est

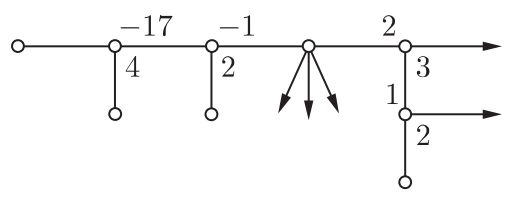

2) Exemple de Chądzyński et Krasiński [CK1] :

$$
f(x, y)=\left(\left(x+y^{q}\right)^{p}+y^{p}\right) .
$$

On a un seul point à l'infini, le point $(1,0,0)$, et

$$
F(1, y, z)=\left(\left(z^{q-1}+y^{q}\right)^{p}+y^{p} z^{p q-1}\right) .
$$

Le germe à l'origine de $F(1, y, z)$ a $p$ branches de paires de Puiseux successives $(q, q-1),(1, q-1)$. Le diagramme associé est

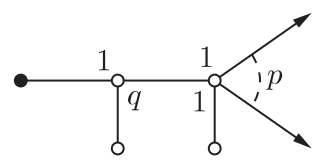

et le diagramme minimal est

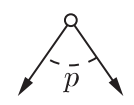

3. Diagrammes minimaux. On note $\Omega_{0}$ le diagramme obtenu en oubliant quel sommet du diagramme minimal est la racine dans le cas où $n_{\infty}>2$, et dans le cas $n_{\infty}=2$ en supprimant la racine et en joignant les deux cotés adjacents en un seul. Neumann a démontré que pour chaque courbe algébrique réduite $\Omega_{0}$ est unique et qu'il y a un nombre fini de manières 
de placer une racine sur $\Omega_{0}$. Si l'on fait cela, on obtient de nouveau un diagramme minimal pour l'entrelacs à l'infini de la courbe $V$ et

ThÉORÈme 3 [N, p. 450]. Pour une courbe algébrique réduite $V \subset$ $\mathbb{C}^{2}$, tout diagramme minimal pour son entrelacs à l'infini est réalisé par un plongement de $\mathbb{C}^{2} \subset \mathbb{P}^{2}$. En particulier, chaque paire $\left(\operatorname{deg}(V), n_{\infty}(V)\right)$ pour $V$ pour laquelle soit $\operatorname{deg}(V)$ est minimal, soit $n_{\infty}(V)>1$ est le degré et le nombre de points à l'infini déterminé par un diagramme minimal pour son entrelacs à l'infini.

Nous allons maintenant rappeler comment on trouve les différentes racines sur $\Omega_{0}$. Tout d'abord, nous enlevons de $\Omega_{0}$ tout coté qui satisfait l'une des deux conditions :

- il existe un poids non positif adjacent au côté,

- il y a deux poids différents de 1 adjacents au côté à l'une de ses extrémités.

Nous notons $\operatorname{rac}\left(\Omega_{0}\right)$ ce qu'il reste de $\Omega_{0}$. Le nombre de façons de mettre une racine sur $\Omega_{0}$ est égal à $1+\sum\left(\delta_{v}-2\right)$, la somme étant prise sur tous les sommets de $\operatorname{rac}\left(\Omega_{0}\right)$ dont seulement 1 poids est supérieur à 1 . Enfin, pour placer les différentes racines, on procède de la manière suivante : on coupe chaque côté de $\operatorname{rac}\left(\Omega_{0}\right)$ en 2 , et on oriente chaque moitié comme suit :

- si un sommet n'a que des poids égaux à 1 , on oriente les demi-côtés à l'opposé du sommet,

- si un sommet a un poids plus grand que les autres, on oriente le demicôté avec poids maximal à l'opposé du sommet, et les autres vers le sommet.

Les racines sont les points d'où partent des flèches.

R e mar que. Il est facile de voir que si l'on fait un automorphisme algébrique linéaire $x=x+a y, y=y$ ou $x=x, y=y+a x$, on ne change pas le diagramme associé à une courbe.

Exemples. 1) Pour l'exemple I, $\operatorname{rac}\left(\Omega_{0}\right)$ est

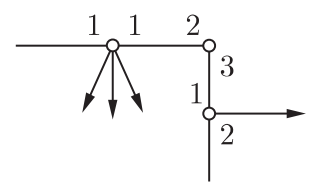

Il y a 2 façons de placer la racine. $\operatorname{rac}\left(\Omega_{0}\right)$ orienté est

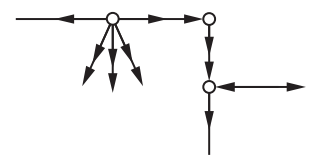


et les deux racines possibles se trouvent

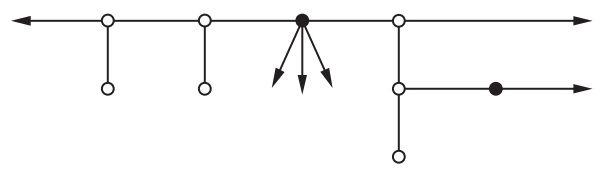

Le diagramme

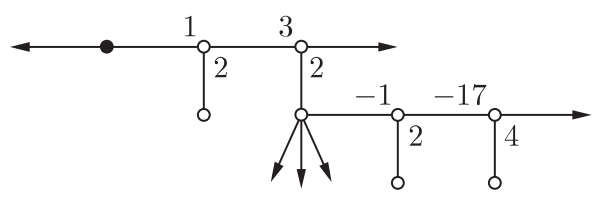

s'obtient avec $g=0$ où $g=f\left(x-y-y^{2}, y\right)$. Il est de degré 27 avec 2 points à l'infini.

II. Nombre de Łojasiewicz à l'infini d'un polynôme. Dans ce paragraphe, nous allons tout d'abord définir le nombre de Łojasiewicz à l'infini d'un polynôme et nous allons montrer comment on peut calculer ce nombre à l'aide des diagrammes de Eisenbud et Neumann des courbes $f(x, y)=t$.

Dans [N], Neumann définit le degré intrinsèque et le nombre de points à l'infini intrinsèque d'une courbe $V$ comme étant le minimum des degrés et le maximum des points à l'infini que l'on peut obtenir avec les plongements $V \subset \mathbb{C}^{2}$. Il montre que le degré intrinsèque s'obtient avec les diagrammes minimaux.

Nous montrons aussi que l'on peut définir un nombre de Lojasiewicz intrinsèque $\mathcal{L}_{\infty \text {,int }}(f)$ à l'infini d'un polynôme.

On montre qu'en général, il n'existe pas pour un polynôme un automorphisme privilégié, c'est-à-dire un automorphisme $\phi$ tel que

$$
\operatorname{deg}(f \circ \phi)=\operatorname{deg}_{\text {int }}(f), \quad n_{\infty}(f \circ \phi)=n_{\infty, \text { int }}(f), \quad \mathcal{L}_{\infty}(f \circ \phi)=\mathcal{L}_{\infty, \text { int }}(f)
$$

mais que c'est vrai sous certaines conditions, en particulier si le polygone de Newton à l'infini de $f \circ \phi$ est non dégénéré.

1. Le nombre de Eojasiewicz à l'infini d'un polynôme. Le nombre de Łojasiewicz à l'infini d'un polynôme $f$ est défini par

$$
\mathcal{L}_{\infty}(f)=\lim _{r \rightarrow \infty} \frac{\ln \phi(r)}{\ln r} \quad \text { où } \quad \phi(r)=\inf _{\|x\|=r}\|\operatorname{grad} f(x)\| .
$$

Nous rappelons tout d'abord, comment, d'après [CK], on peut calculer le nombre de Łojasiewicz à l'infini d'un polynôme. 
Soit $X=\{t \in \mathbb{C}:|t|>E\}$. Une fonction $h: X \rightarrow \mathbb{C}^{2}$ est dite méromorphe à l'infini $[\mathrm{CK}]$ si elle peut être représentée par une série de Laurent de la forme

$$
h(y)=c_{k} y^{k}+\ldots+c_{0}+c_{-1}(1 / y)+\ldots
$$

convergente dans $X$. Si $h \neq 0$, alors le plus grand indice pour lequel $c_{i} \neq 0$ est appelé le degré de $h$. Si $h=0$ on définit $\operatorname{deg}(h)=-\infty$. Soit maintenant $H=\left(h_{1}, h_{2}\right)$ une paire de fonctions méromorphes définies sur $X$. On définit $\operatorname{deg}(H)=\max \left(\operatorname{deg}\left(h_{1}\right), \operatorname{deg}\left(h_{2}\right)\right)$. On dit qu'une application méromorphe $H$ admet un pôle à l'infini si au moins une des deux fonctions $h_{1}, h_{2}$ a un pôle à l'infini. On a le résultat suivant :

Lemme 4 [CK, p. 148]. Soit $h: \mathbb{C}^{2} \rightarrow \mathbb{C}$ une fonction polynomiale non constante, et $V \subset \mathbb{C}^{2}$ la courbe définie par $h(x, y)=0$. Alors il existe un voisinage $Y$ de l'infini dans $\mathbb{C}^{2}$ tel que $V \cap Y$ est la réunion de $r$ composantes, chacune d'elles étant homéomorphe à un sous-ensemble $X=\left\{t \in \mathbb{C}^{2}\right.$ : $|t|>g\}$; l'homéomorphisme est défini par l'application $\psi: X \rightarrow Y$ méromorphe à l'infini et $y$ ayant un pôle.

Les composantes de $V \cap Y$ sont appelées les branches de la courbe $h=0$ dans le voisinage $Y$ et $(X, \psi, Y)$ leur paramétrisation.

Soient $\left\{\left(X_{i}, a_{i}, Y\right): i=1, \ldots, r\right\}$ l'ensemble des branches de la courbe $f_{x}(x, y)=0$ et $\left\{\left(X_{j}^{\prime}, b_{j}, Y\right): j=1, \ldots, s\right\}$ l'ensemble des branches de la courbe $f_{y}(x, y)=0$.

Lemme 5 [CK, Main Theorem]. On a

$$
\mathcal{L}_{\infty}(f)=\min _{i, j}\left(\frac{\operatorname{deg}\left(f_{y}^{\prime} \circ a_{i}\right)}{\operatorname{deg}\left(a_{i}\right)}, \frac{\operatorname{deg}\left(f_{x}^{\prime} \circ b_{j}\right)}{\operatorname{deg}\left(b_{j}\right)}\right) .
$$

EXEMPLE. On considère

$$
f_{t}=y\left(x y^{3}+1\right)^{2}+x^{6}+t .
$$

Alors $f_{x}^{\prime}$ a deux branches, $a_{1}=\left(-1 / u^{3}+3 / u^{22}+\ldots, u\right)$ et $a_{2}=\left(u^{7}+\right.$ $\left.\ldots, c u^{4}+\ldots\right)$ où $c^{7}+3=0$, et

$$
\frac{\operatorname{deg}\left(f_{y}^{\prime} \circ a_{1}\right)}{\operatorname{deg}\left(a_{1}\right)}=-19, \quad \frac{\operatorname{deg}\left(f_{y}^{\prime} \circ a_{2}\right)}{\operatorname{deg}\left(a_{2}\right)}=\frac{38}{7} ;
$$

$f_{y}^{\prime}$ a quatre branches, $b_{1}=\left(-1 / u^{3}, u\right), b_{2}=\left(-7 / u^{3}, u\right), b_{3}=\left(u^{3},-1 / u\right)$, $b_{4}=\left(u^{3},-7 / u\right)$, et

$$
\begin{array}{ll}
\frac{\operatorname{deg}\left(f_{x}^{\prime} \circ b_{1}\right)}{\operatorname{deg}\left(b_{1}\right)}=-15, & \frac{\operatorname{deg}\left(f_{x}^{\prime} \circ b_{2}\right)}{\operatorname{deg}\left(b_{2}\right)}=4, \\
\frac{\operatorname{deg}\left(f_{x}^{\prime} \circ b_{3}\right)}{\operatorname{deg}\left(b_{3}\right)}=5, & \frac{\operatorname{deg}\left(f_{x}^{\prime} \circ b_{4}\right)}{\operatorname{deg}\left(b_{4}\right)}=5 .
\end{array}
$$


On a donc

$$
\mathcal{L}_{\infty}(f)=-19 .
$$

Nous montrons maintenant comment on peut calculer $\mathcal{L}_{\infty}(f)$ à l'aide des diagrammes de Eisenbud et Neumann de toutes les courbes définies par $f(x, y)=t$.

On note $\mathcal{L}_{t}$ le diagramme de Eisenbud et Neumann de la courbe $f(x, y)$ $=t$. A chaque sommet $v \in \mathcal{V}_{\mathcal{L}_{t}}$ on associe sa multiplicité $l_{t, v}$ et son "braid index", $\varrho_{t, v}$, qui est le nombre d'enlacement de la composante virtuelle associée au sommet $v$ et de la composante virtuelle associée à la racine.

Proposition 6. On a

$$
\mathcal{L}_{\infty}(f)=\inf _{t \in \mathbb{C}} \inf _{v \in \mathcal{V}_{\mathcal{L}_{t}}} \frac{l_{t, v}}{\varrho_{t, v}}-1 .
$$

Preuve. On note $F(x, y, z)$ le polynôme homogène associé à $f(x, y)$. Supposons que $x=A_{1}(v), z=A_{2}(v)$ soit un développement de Puiseux de $F_{x}^{\prime}(x, 1, z)$ au voisinage d'un point singulier. Alors une paramétrisation de $f_{x}^{\prime}(x, y)$ est donnée par $x=a_{1}(u), y=a_{2}(u)$ où

$$
a_{1}(u)=\frac{A_{1}(1 / u)}{A_{2}(1 / u)}, \quad a_{2}(u)=\frac{1}{A_{2}(1 / u)} .
$$

De plus, si

$$
F_{x}^{\prime}\left(A_{1}(v), 1, A_{2}(v)\right)=a_{0} v^{a}+a_{1} v^{a+1}+\ldots,
$$

on a

$$
F_{x}^{\prime}\left(\frac{A_{1}(v)}{A_{2}(v)}, \frac{1}{A_{2}(v)}, 1\right)=\left(\frac{1}{A_{2}(v)}\right)^{d-1}\left(a_{0} v^{a}+a_{1} v^{a+1}+\ldots\right)
$$

où $d$ est le degré de $f$ et

$$
f_{x}^{\prime}\left(a_{1}(u), a_{2}(u)\right)=\left(a_{2}(u)\right)^{d-1}\left(a_{0} u^{-a}+a_{1} u^{-(a+1)}+\ldots\right) .
$$

Soit

$$
F_{t}(x, y, z)=F(x, y, z)-t z^{d} .
$$

On considère le germe analytique $F_{t}(x, 1, z)$ au point $\left(\alpha_{q}, \beta_{q}, 0\right)$. Soit $S$ la polaire dans la direction $z=0$. Alors $S$ est définie par $F_{x}^{\prime}(x, 1, z)=0$. Soit $\gamma_{i}$ une branche de $S$. On suppose $\gamma_{i}$ paramétrée par $x=A_{i, 1}(v), y=A_{i, 2}(v)=$ $v^{a_{i}}+\ldots$ On écrit

$$
\begin{aligned}
F_{t}\left(\gamma_{i}\right) & =B v^{e_{i}(t)}+\ldots, \\
\frac{d}{d v} F_{t}\left(\gamma_{i}\right) & =A_{i, 1}^{\prime}(v) F_{x}^{\prime}\left(\gamma_{i}\right)+A_{i, 2}^{\prime}(v) F_{z}^{\prime}\left(\gamma_{i}\right) .
\end{aligned}
$$

Donc

$$
F_{z}^{\prime}\left(\gamma_{i}\right)=\frac{B e_{i}(t)}{a_{i}} v^{e_{i}(t)-a_{i}}+\ldots
$$


Or

$$
\begin{aligned}
d F_{t} & =x F_{x}^{\prime}+F_{y}^{\prime}+z F_{z}^{\prime}, \\
F_{y}^{\prime}\left(\gamma_{i}\right) & =\left(d B-\frac{B e_{i}(t)}{a_{i}}\right) v^{e_{i}(t)}+\ldots=B\left(d-\frac{e_{i}(t)}{a_{i}}\right) v^{e_{i}(t)}+\ldots
\end{aligned}
$$

On en déduit

$$
f_{y}^{\prime}\left(a_{i, 1}(u), a_{i, 2}(u)\right)=B\left(d-\frac{e_{i}(t)}{a_{i}}\right) u^{a_{i}(d-1)-e_{i}(t)}+\ldots
$$

On écrit

$$
f\left(a_{i, 1}(u), a_{i, 2}(u)\right)=A_{i} u^{e_{i}}+\ldots
$$

Alors, $e_{i}(t)=\inf \left(d a_{i}-e_{i}, d a_{i}\right)$. Si $\gamma_{i}$ est telle que $e_{i}>0$, alors, pour tout $t$, $e_{i}(t)=\left(d a_{i}-e_{i}\right)$, et si $\gamma_{i}$ est telle que $e_{i} \leq 0$, on a $e_{i}(t)=d a_{i}$ si $t \neq A_{i}$ et $e_{i}(t)<d a_{i}$ si $t=A_{i}$.

Donc,

$$
\operatorname{deg}\left(f_{y}^{\prime}\left(a_{i, 1}(u), a_{i, 2}(u)\right)=a_{i}(d-1)-e_{i}(t)\right.
$$

avec $t$ quelconque si $\gamma_{i}$ est telle que $e_{i}>0$, et $t=A_{i}$ si $\gamma_{i}$ est telle que $e_{i} \leq 0$.

On fait un changement de variables linéaire, de telle sorte que les points à l'infini de $f$ soient de la forme $\left(\alpha_{q}, \beta_{q}, 1\right)$ avec $\alpha_{q} \neq 0$ et $\beta_{q} \neq 0$. Dans ce cas

$$
\operatorname{deg}\left(a_{i, 1}(u), a_{i, 2}(u)\right)=\operatorname{deg}\left(a_{i, 2}(u)\right)
$$

Alors

$$
\frac{\operatorname{deg}\left(f_{y}^{\prime}\left(a_{i, 1}(u), a_{i, 2}(u)\right)\right)}{\left.\operatorname{deg}\left(a_{i, 2}(u)\right)\right)}=d-1-\frac{e_{i}(t)}{a_{i}}
$$

avec $t$ quelconque si $\gamma_{i}$ est telle que $e_{i}<0$, et $t=A_{i}$ si $\gamma_{i}$ est telle que $e_{i} \geq 0$. D'après [T], pour $t$ fixé l'ensemble $\left\{e_{i}(t) / a_{i}+1: i=1, \ldots, r\right\}$ est l'ensemble des invariants polaires du germe analytique $F_{t}(x, 1, z)$ au point $\left(\alpha_{q}, \beta_{q}\right)$. Notons $\mathcal{L}_{q, t}$ le diagramme de Eisenbud et Neumann local du germe analytique $F_{t}(x, 1, z)$ au point $\left(\alpha_{q}, \beta_{q}\right)$ et $l_{v, t}^{q}\left(\right.$ resp. $\left.\varrho_{v, t}^{q}\right)$ la multiplicité (resp. le "braid index") du sommet $v \in \mathcal{V}_{\mathcal{L}_{q, t}}$. Alors d'après [LMW],

$$
E=\left\{\frac{e_{i}(t)}{a_{i}}+1: i=1, \ldots, r\right\}=\left\{\frac{l_{v, t}^{q}}{\varrho_{v, t}^{q}}-1: v \in \mathcal{V}_{\mathcal{L}_{q, t}}\right\} \cup\{\min (E)\}
$$

Le lien entre $l_{v, t}^{q}$ pour $v \in \mathcal{V}_{\mathcal{L}_{q, t}}$ et $l_{v, t}$ pour le sommet correspondant de $\mathcal{V}_{\mathcal{L}_{t}}$ est donné par [LN, Lemme 2.1]

$$
l_{v, t}=d \varrho_{v, t}-l_{v, t}^{q} .
$$

On en déduit donc la proposition. 
Remarque. Dans $[\mathrm{H}]$, on définit le nombre de Eojasiewicz à l'infini d'une courbe par

où

$$
\mathcal{L}_{\infty, t}(f)=\lim _{\delta \rightarrow 0} \lim _{r \rightarrow \infty} \frac{\ln \phi_{\delta}(r)}{\ln r}
$$

$$
\phi_{\delta}(r)=\inf _{\|x\|=r,\left|f(x)-t_{0}\right| \leq \delta}\|\operatorname{grad} f(x)\|,
$$

et on montre que si $\mathcal{L}_{\infty, t}(f)<0$,

$$
\mathcal{L}_{\infty, t}(f)=\min _{i, j}\left(\frac{\operatorname{deg}\left(f \circ a_{i}-t\right)}{\operatorname{deg}\left(a_{i}\right)}, \frac{\operatorname{deg}\left(f \circ b_{j}-t\right)}{\operatorname{deg}\left(b_{j}\right)}\right)-1 .
$$

Exemple. Dans l'exemple précédent, on a

$$
\begin{array}{cl}
\frac{\operatorname{deg}\left(f_{t} \circ a_{1}\right)}{\operatorname{deg}\left(a_{1}\right)}=0 \quad \text { si } t \neq 0, & \frac{\operatorname{deg}\left(f_{0} \circ a_{1}\right)}{\operatorname{deg}\left(a_{1}\right)}=-18, \\
\frac{\operatorname{deg}\left(f_{t} \circ a_{2}\right)}{\operatorname{deg}\left(a_{2}\right)}=6 & \text { pour tout } t .
\end{array}
$$

De plus,

et

$$
\frac{\operatorname{deg}\left(f_{t} \circ b_{1}\right)}{\operatorname{deg}\left(b_{1}\right)}=0 \quad \text { si } t \neq 0, \quad \frac{\operatorname{deg}\left(f_{0} \circ b_{1}\right)}{\operatorname{deg}\left(b_{1}\right)}=-18,
$$

$$
\frac{\operatorname{deg}\left(f_{t} \circ b_{2}\right)}{\operatorname{deg}\left(b_{2}\right)}=1, \quad \frac{\operatorname{deg}\left(f_{t} \circ b_{3}\right)}{\operatorname{deg}\left(b_{3}\right)}=6, \quad \frac{\operatorname{deg}\left(f_{t} \circ b_{4}\right)}{\operatorname{deg}\left(b_{4}\right)}=6
$$

pour tout $t$. Donc $\mathcal{L}_{\infty, t}(f)=-1$ si $t \neq 0$, et $\mathcal{L}_{\infty, 0}(f)=-19$.

En utilisant [L, Theorem 4.1], on voit que si $\mathcal{L}_{\infty, t}(f)<0$,

et donc

$$
\mathcal{L}_{\infty, t}(f)=\inf _{v \in \mathcal{V}_{\mathcal{L}_{t}}} \frac{l_{v, t}}{\varrho_{v, t}}-1
$$

$$
\mathcal{L}_{\infty}(f)=\inf _{t \in \mathbb{C}} \mathcal{L}_{\infty, t}(f) .
$$

2. Le nombre de Eojasiewicz à l'infini intrinsèque d'un polynôme. Nous allons montrer que l'on peut aussi définir un nombre de Łojasiewicz à l'infini intrinsèque.

Proposition 7. Soit $V$ une courbe algébrique réduite, $\mathcal{L}$ son diagramme associé et $\mathcal{L}_{\min }$ son diagramme minimal associé. Alors

$$
\inf _{v \in \mathcal{V}_{L}} \frac{\left|l_{v}\right|}{\varrho_{v}} \leq \inf _{v \in \mathcal{V}_{L_{\min }}} \frac{\left|l_{v}\right|}{\varrho_{v}} .
$$

D'après [CN, Lemme 9, p. 297], $\inf _{v \in \mathcal{V}_{L}}\left|l_{v}\right| / \varrho_{v}$ est obtenu avec un sommet voisin d'une flèche, et dans ce cas $\varrho_{v}$ est la multiplicité de la branche 
analytique qui correspond à la flèche, c'est-à-dire le produit de tous les nombres qui sont adjacents au chemin qui va de la flèche à la racine.

En rendant le diagramme minimal, on supprime tout côté de la forme

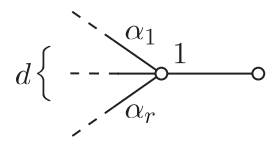

Si $d \geq 3$, on remplace

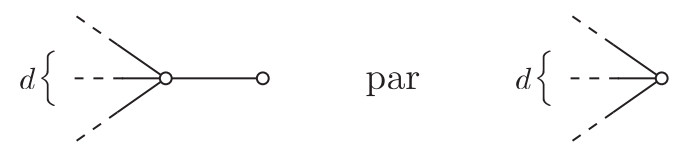

On ne change donc aucun des $l_{v}$ ni des $\varrho_{v}$.

Si $d=2$, on passe de

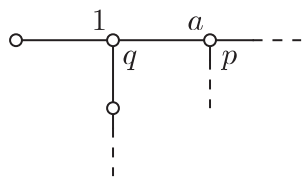

à

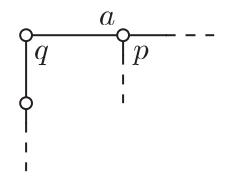

et ici on supprime un sommet de valence 2 :

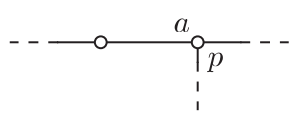

Dans cette opération, il est possible que certains $\varrho_{v}$ soient divisés par $q$ mais les $l_{v}$ ne changent pas.

Enfin, on considère

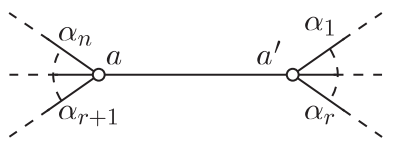

On sait que l'on a sur chaque sommet au plus 2 poids qui sont supérieurs à 1 , et ils sont premiers entre eux. Si

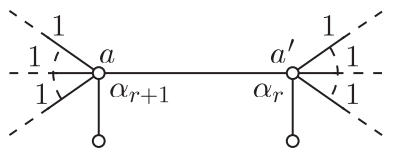


on a $a a^{\prime}=\alpha_{r} \alpha_{r+1}$. D'après le lemme de Gauss, $a=\alpha_{r}$ et $a^{\prime}=\alpha_{r+1}$. On obtient

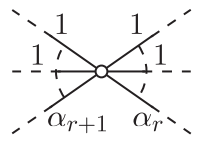

Dans cette opération $\varrho_{v}$ peut être divisé par $\alpha_{r}$ ou $\alpha_{r+1}$ ou le produit. Dans le calcul des $l_{v}, a^{\prime}$ ou $a$ peuvent être remplacés par $\alpha_{r+1}$ ou $\alpha_{r}$, ce qui ne change pas $l_{v}$. La proposition est donc démontrée.

R e mar que. Dans le passage de $\mathcal{L}$ à $\mathcal{L}_{\text {min }}$, le nombre de points à l'infini ne peut lui aussi qu'augmenter. En effet, si

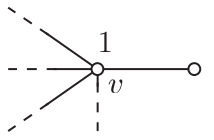

alors le sommet $v$ ne peut être la racine de $\mathcal{L}$, car tout côté qui part de la racine doit aboutir à une flèche.

Si l'on supprime un sommet de valence 2, on ne peut faire baisser le nombre de points à l'infini que si le diagramme minimal est

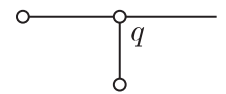

avec $p>1$, mais dans ce cas la racine ne pouvait pas se trouver ailleurs car le poids d'un sommet qui n'est pas du côté de la racine par rapport à ce sommet est égal à 1 . La troisième opération pour rendre les diagrammes minimaux augmente éventuellement la valence de la racine.

On dit qu'un polynôme est bon si toutes les courbes $f(x, y)=t$ sont régulières. Dans ce cas, les diagrammes associés à toutes les courbes $f(x, y)$ $=t$ sont les mêmes, il y a un nombre fini de diagrammes minimaux et on peut définir

$$
\mathcal{L}_{\infty, \text { int }}(f)=\max _{L_{\min }} \inf _{v \in \mathcal{V}_{L_{\min }}} \frac{l_{v}}{\varrho_{v}}-1
$$

où $L_{\min }$ est un diagramme associé à la courbe générique.

Si le polynôme $f$ n'est pas bon, on considère tous les diagrammes associés aux courbes $f(x, y)=t$. Il y en a un nombre fini, celui de la courbe générique, qui est régulière, et celui des courbes spéciales. On considère maintenant le diagramme minimal associé à la courbe générique. L'automorphisme $\phi$ 
correspondant ne donne pas nécéssairement un diagramme minimal pour les courbes spéciales, mais on peut montrer que

$$
\inf _{L_{t, \phi, \min }} \inf _{v \in \mathcal{V}_{L_{t, \phi} \text { min }}} \frac{l_{t, v}}{\varrho_{t, v}}=\inf _{v \in \mathcal{V}_{L_{t, \phi}}} \frac{l_{t, v}}{\varrho_{t, v}}
$$

où $L_{t, \phi}$ désigne le diagramme associé à $f \circ \phi-t$, et $L_{t, \phi, \text { min }}$ les diagrammes minimaux associés. En effet, d'après [LN], on sait comment reconstituer le diagramme de Eisenbud et Neumann de la courbe générique à partir des diagrammes de Eisenbud et Neumann des courbes spéciales. Donc, pour passer de $L_{t, \phi}$ à $L_{t, \phi, \min }$, on ne doit pas changer le degré. Or le passage d'un diagramme à un diagramme minimal ne peut que diminuer les multiplicités des branches locales. Si le degré ne bouge pas, aucune des multiplicités locales des branches ne change, c'est-à-dire, le "braid index" des sommets adjacents aux flèches ne change pas.

On peut donc définir, si $f$ n'est pas bon,

$$
\mathcal{L}_{\infty, \text { int }}(f)=\inf _{t \in \mathbb{C}} \inf _{L_{t, \min }} \inf _{v \in \mathcal{V}_{L_{t, \min }}} \frac{l_{v}}{\varrho_{v}}-1
$$

Re marque. Si le polynôme $f$ est bon, alors pour tout automorphisme $\phi, f \circ \phi$ est bon. En effet, d'après [H], $f$ est bon si et seulement si $\mathcal{L}_{\infty}(f) \geq$ -1 . Ceci est équivalent à $l_{v} \geq 0$ pour tout $v \in \mathcal{V}$ pour le diagramme minimal associé à $f$, et c'est encore équivalent à $l_{v} \geq 0$ pour tout $v \in \mathcal{V}$ pour tous les diagrammes associés à $f \circ \phi$. Donc $f \circ \phi$ est bon.

Dans tous les cas, on a

THÉORÈme 8. Si $f$ est bon,

$$
\mathcal{L}_{\infty, \text { int }}(f)=\max _{\phi \in \operatorname{Aut}\left(\mathbb{C}^{2}\right)} \mathcal{L}_{\infty}(f \circ \phi) .
$$

Si f n'est pas bon,

$$
\mathcal{L}_{\infty, \text { int }}(f)=\min _{\phi \in \operatorname{Aut}\left(\mathbb{C}^{2}\right)} \mathcal{L}_{\infty}(f \circ \phi) .
$$

3. Existence d'un automorphisme privilégié pour un polynôme. On peut maintenant se poser le problème suivant :

Existe-t-il un automorphisme $\phi \in \mathbb{C}^{2}$ tel que $\operatorname{deg}(f \circ \phi)=\operatorname{deg}_{\text {int }}(f)$, $n_{\infty}(f \circ \phi)=n_{\infty, \text { int }}(f)$ et $\mathcal{L}_{\infty}(f \circ \phi)=\mathcal{L}_{\infty, \text { int }}(f)$ ?

La réponse est non en général, comme le montre l'exemple suivant :

EXEMPLE. On considère la courbe $V$ définie par $f(x, y)=0$ où

$$
\begin{aligned}
f(x, y)= & \left(x^{3}+y\right)^{7} y^{8}+x^{25} y^{4}+x^{18}-20 x^{2} y^{8}\left(x^{3}+y\right)^{5} \\
& -64 x y^{12}-177 x^{4} y^{11}-157 x^{7} y^{10}-43 x^{10} y^{9} \\
& -33 x^{2} y^{11}+41 x^{5} y^{10}+200 x^{8} y^{9}+148 x^{11} y^{8}+25 x^{14} y^{7} \\
& +3 x^{17} y^{6}-72 y^{11}-233 x^{3} y^{10}-206 x^{6} y^{9}-45 x^{9} y^{8} .
\end{aligned}
$$


Alors le diagramme de Eisenbud et Neumann associé est

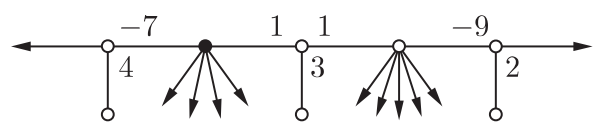

On a $\operatorname{deg}(f)=29, n_{\infty}(f)=6$ et $\mathcal{L}_{\infty}(f)=20 / 3$.

On a 2 façons de mettre la racine,

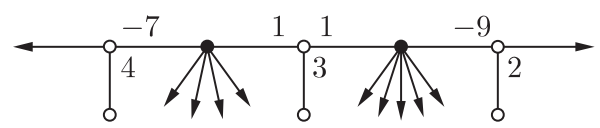

et pour le diagramme

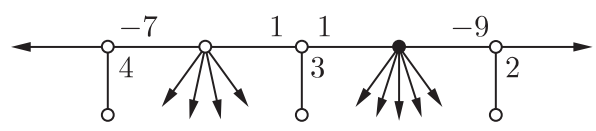

on a $\operatorname{deg}(g)=31, n_{\infty}(g)=7$ et $\mathcal{L}_{\infty}(g)=6$.

On va étudier quelques cas où l'on a un plongement privilégié. Si

$$
f(x, y)=\sum a_{\gamma} x^{\gamma_{1}} y^{\gamma_{2}}
$$

on appelle polygone de Newton de $f$, noté $\Delta(f)$, l'enveloppe convexe de l'ensemble

$$
\operatorname{Supp} f=\left\{\gamma: a_{\gamma} \neq 0\right\} \text {. }
$$

On note $\Delta_{\infty}(f)=\Delta(f)-\left(\Delta_{0}(f) \cap \Delta(f)\right)$ où $\Delta_{0}(f)$ désigne le polygone de Newton à l'origine de $f$. On appelle $\Delta_{\infty}(f)$ le polygone de Newton à l'infini de $f$.

ThÉORÈme 9. Soit $f$ un polynôme. Alors si l'on a l'une des trois propriétés suivantes :

(i) $n_{\infty}(f)=1$,

(ii) le polygone de Newton à l'infini de $f$ est contenu dans un rectangle dont les côtés sont parallèles aux axes, un sommet à l'origine et le sommet opposé sur la droite $x+y=\operatorname{deg}(f)$,

(iii) le polygone de Newton à l'infini de $f$ est non dégénéré, on a $\operatorname{deg}(f)=\operatorname{deg}_{\text {int }}(f), n_{\infty}(f)=n_{\infty, \text { int }}(f)$ et $\mathcal{L}_{\infty}(f)=\mathcal{L}_{\infty, \text { int }}(f)$.

Preuve. Dans le cas (i), on a déjà remarqué qu'il n'y a qu'un seul diagramme minimal. 
Il en est de même dans le cas (ii). En effet, sur chaque côté qui part de la racine, le poids à l'autre extrémité est négatif ou nul, car les premières paires de Puiseux sont données par les pentes du polygone de Newton de $f$ [CN]. Maintenant si l'on a

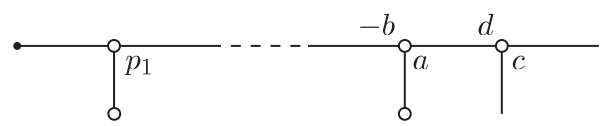

l'entrelacs algébrique correspondant vérifie

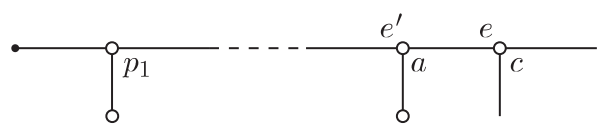

où $e=a^{2} c \prod_{i} p_{i}^{2}-d$ et $e^{\prime}=a \prod_{i} p_{i}^{2}+b$. Mais comme $e>a c e^{\prime}$, on en déduit que $d<0$. Donc $\operatorname{rac}\left(\Omega_{0}\right)$ ne contient qu'un seul côté et il n'y a qu'une seule façon de mettre la racine. On peut remarquer que les polynômes qui satisfont l'hypothèse du jacobien vérifient cette propriété $[\mathrm{O}]$.

Dans la cas (iii), nous supposons que nous ne sommes pas dans les cas (i) et (ii). Nous allons tout d'abord montrer que les autres possibilités de placer la racine donnent $n_{\infty}=2$.

On note $d=\operatorname{deg}(f)$. On note $\sigma_{0}$ la face de $\Delta_{\infty}(f)$ d'équation $x+y=d$, et $\alpha^{1}=\left(\alpha_{1}^{1}, \alpha_{2}^{1}\right), \alpha^{2}=\left(\alpha_{1}^{2}, \alpha_{2}^{2}\right)$ les sommets de $\Delta_{\infty}(f)$ qui sont sur cette face, avec $\alpha_{1}^{1} \leq \alpha_{2}^{1}$. On note $\Delta_{\infty}^{\prime}(f)$ la partie de $\Delta_{\infty}(f)$ qui se trouve au-dessus de la droite passant par l'origine et $\alpha^{1}$ et $\Delta_{\infty}^{\prime \prime}(f)$ la partie de $\Delta_{\infty}(f)$ qui se trouve en dessous de la droite qui passe par l'origine et $\alpha^{2}$ :

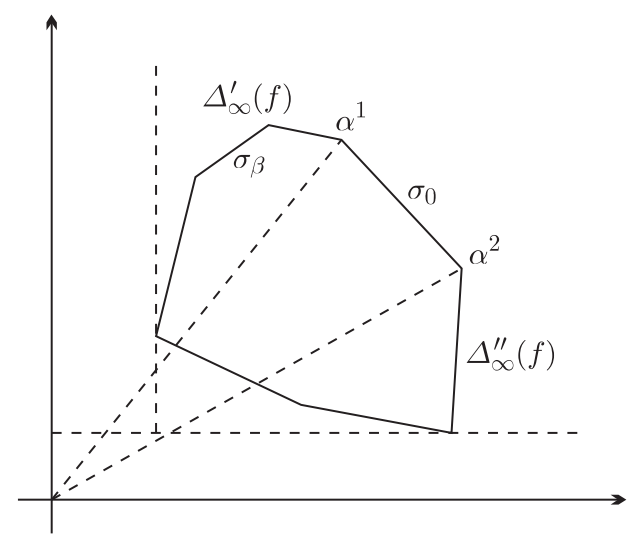

Chaque sommet du diagramme de Eisenbud et Neumann correspond à une 
face de $\Delta_{\infty}(f)$; si l'on écrit l'équation de $\sigma_{\beta}$, dont les sommets sont $\beta^{1}$ et $\beta^{2}$,

$$
\frac{q_{\beta} x+p_{\beta} y}{\Delta_{\beta}}=1
$$

$\mathrm{Ou}$

$$
\frac{q_{\beta} y+p_{\beta} x}{\Delta_{\beta}}=1 \quad \text { avec } p_{\beta}>0,\left(p_{\beta}, q_{\beta}\right)=1,
$$

le sommet corespondant a pour poids $p_{\beta}, q_{\beta}$, et on a $l_{\beta}=\Delta_{\beta}$. Le nombre de flèches qui partent du sommet est $k_{\beta}=\operatorname{pgcd}\left(\beta_{1}^{2}-\beta_{1}^{1}, \beta_{2}^{2}-\beta_{2}^{1}\right)$. Pour qu'il existe une autre racine pour le diagramme minimal, il est nécessaire et suffisant qu'il existe un sommet dont un seul des poids soit supérieur à 1 . D'après la correspondance entre les sommets du diagramme et les faces du polygone de Newton, le seul sommet dont tous les poids sont 1 est celui de $\sigma_{0}$. Or un sommet ne peut être une racine que si tous ses poids sont 1 . Donc toutes les autres racines du diagramme sont de valence 2 .

Maintenant nous montrons que le degré minimum est obtenu avec $d$. On a

$$
d=k_{0}+n_{1}+n_{2}
$$

où $n_{1}$ et $n_{2}$ sont des degrés par rapport à chacune des indéterminées $x, y$. De plus,

$$
n_{1}=p_{1} k_{1}+p_{2} k_{2}+\ldots+p_{m_{1}} k_{m_{1}}+\varepsilon_{1}
$$

où $\varepsilon_{1}$ vaut 0 si $\Delta_{\infty}(f)$ rencontre l'axe des $x$ et 1 sinon. (On suppose que les singularités sont isolées.) Supposons maintenant que nous ayons une racine sur une flèche partant du sommet $\beta_{i}$ correspondant à une face $\sigma_{i}$ de $\Delta_{\infty}^{\prime \prime}(f)$ :

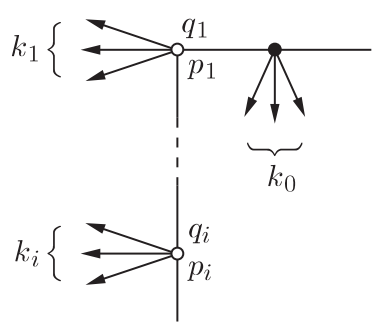

Alors le degré correspondant est égal à

$$
\begin{aligned}
d_{i}= & +p_{i}\left(k_{i}-1\right)+p_{i+1} k_{i+1}+\ldots+p_{m_{1}} k_{m_{1}}+\varepsilon_{1} \\
& +p_{i}\left(q_{i-1} k_{i-1}+\ldots+q_{1} k_{1}+k_{0}+n_{2}\right) .
\end{aligned}
$$

Or on a $p_{i} q_{i-1}>p_{i-1}$. Donc

$$
d_{i}>1+n_{1}+p_{i} k_{0}+p_{i} n_{i}-p_{i} .
$$


Si $k_{0}=0$ et $n_{2}=1$, alors $d_{i}>n_{1}+1=d$; si $k_{0}>0$ et $n_{2}=1$, alors $d_{i}>n_{1}+1+k_{0}=d$; enfin si $k_{0}>0$ et $n_{2}>1$, alors $d_{i}>n_{1}+n_{2}+k_{0}+$ $p_{i} k_{0}-k_{0}-p_{i}+1=d+\left(p_{i}-1\right)\left(k_{0}-1\right)$.

Il nous reste maintenant à montrer que $\mathcal{L}_{\infty}=\mathcal{L}_{\infty, \text { int }}$. Cette propriété est une conséquence de la proposition suivante qui est l'analogue à l'infini du théorème de Lichtin [Li].

Proposition 10. Si le polygone de Newton à l'infini de $f$ est non dégénéré, alors $\mathcal{L}_{\infty}(f)$ est égal au minimum des abscisses ou ordonnées des intersections du polygone de Newton de $f$ avec les axes.

Nous poursuivons la démonstration du théorème 9 en utilisant la proposition 10.

Si l'on suppose que l'on a une racine sur une flèche qui part du sommet $\beta_{i}$, on sait, d'après [CN, Lemme 9], que $l_{v} / \varrho_{v}$ diminue quand on s'éloigne de la racine :

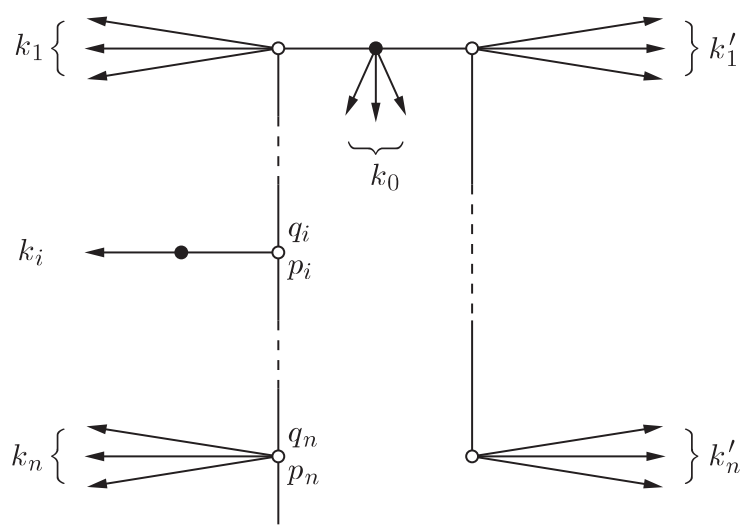

Alors, en changeant de racine, soit le nombre de Łojasiewicz est inchangé, soit il est divisé par $p_{i}$.

EXEMPLE. Soit

$$
f(x, y)=x^{8}+x^{7} y^{2}+x^{5} y^{5}+x^{3} y^{7}+y^{8} .
$$

Le diagramme de l'entrelacs à l'infini de $f(x, y)=0$ est

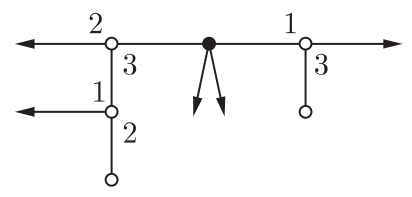

On a $n_{\infty}=4, d=10, \mathcal{L}_{\infty}=8$. Il y a 3 façons de mettre la racine : 


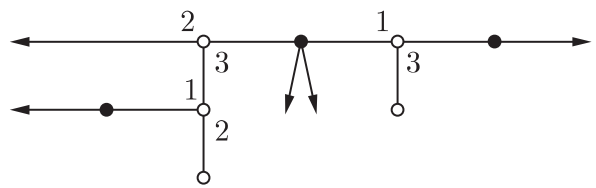

$\mathrm{Si}$

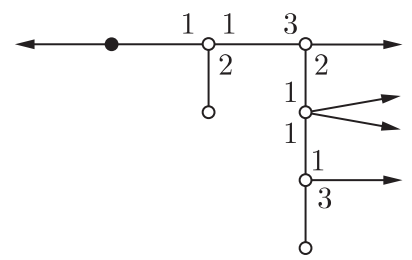

on a l'entrelacs à l'infini de $g(x, y)=f\left(x-y-y^{2}, y\right)$, et $n_{\infty}=2, d=15$ et $\mathcal{L}_{\infty}=8 / 3$.

$\mathrm{Si}$

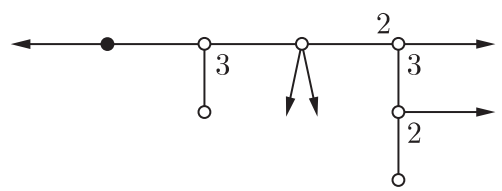

on a l'entrelacs à l'infini de $g(x, y)=f\left(x, y-x^{3}\right)$, et $n_{\infty}=2, d=22$ et $\mathcal{L}_{\infty}=8 / 3$.

Il nous reste maintenant à démontrer la Proposition 10.

Preuve de la Proposition 10. Nous avons remarqué que $l_{v_{\beta}}=$ $\Delta_{\beta}$, lorsque l'équation de $\sigma_{\beta}$ est

$$
\frac{q_{\beta} x+p_{\beta} y}{\Delta_{\beta}}=1 \quad \text { ou } \quad \frac{q_{\beta} y+p_{\beta} x}{\Delta_{\beta}}=1 .
$$

Donc $l_{v_{\beta}} / p_{\beta}=l_{v_{\beta}} / \varrho_{v_{\beta}}$ est l'abscisse ou l'ordonnée de l'intersection de la face $\sigma_{\beta}$ avec les axes. Donc la Proposition 10 est démontrée.

Cet article a été écrit lors d'un séjour du deuxième auteur à l'Université Bordeaux I, comme professeur invité pendant un mois. Les auteurs remercient l'Université pour leur avoir ainsi permis de mener à bien ce travail.

\section{Références}

[CN] P. Cassou-Noguès, Entrelacs toriques itérés et intégrales associées à une courbe plane, Sém. Théorie de Nombres Bordeaux 2 (1990), 237-331. 
[CK] J. Chạdzyński and T. Krasiński, Exponent of growth of polynomial mappings of $\mathbb{C}^{2}$ into $\mathbb{C}^{2}$, in: Singularities, Banach Center Publ. 20, PWN, Warszawa, 1988, $147-160$.

[CK1] J. Chạdzyński and T. Krasiński, Sur l'exposant de Eojasiewicz à l'infini pour les applications polynomiales de $\mathbb{C}^{2}$ dans $\mathbb{C}^{2}$ et les composantes des automorphismes polynomiaux de $\mathbb{C}^{2}$, C. R. Acad. Sci. Paris Sér. I 315 (1992), 1399-1402.

[EN] D. Eisenbud and W. D. Neumann, Three-Dimensional Link Theory and Invariants of Plane Curve Singularities, Ann. of Math. Stud. 101, Princeton Univ. Press, Princeton, N.J., 1985.

[H] Ha Huy Vui, On the irregular at infinity algebraic plane curves, preprint, Institute of Mathematics, National Center for Scientific Research of Vietnam, 1991.

[LMW] Le Dung Trang, F. Michel et C. Weber, Sur le comportement des polaires associées aux germes de courbes planes, Compositio Math. 72 (1989), 87-113.

[L] Le Van Thanh, Affine polar quotients of algebraic plane curves, Acta Math. Vietnam. 17 (1992), 95-102.

[LN] Le Van Thanh and W. Neumann, On irregular links at infinity of algebraic plane curves, Math. Ann., to appear.

[Li] B. Lichtin, Estimations of Eojasiewicz exponents and Newton polygons, Invent. Math. 64 (1981), 417-429.

[N] W. D. Neumann, Complex algebraic plane curves via their links at infinity, ibid. 98 (1989), 445-489.

[O] M. Oka, On the boundary to the Jacobian problem, Kodai Math. J. 6 (1983), 419-433.

[T] B. Teissier, Variétés polaires, Invent. Math. 41 (1977), 103-111.

CEREMAB, UNIVERSITÉ BORDEAUX I

350, COURS DE LA LIBÉRATION

33405 TALENCE CEDEX 05, FRANCE

E-mail: CASSOU@CEREMAB.U-BORDEAUX.FR
INSTITUTE OF MATHEMATICS

P.O. BOX 601

BO HO

10000 HANOI, VIETNAM 